Revisión

\title{
Revisión del tratamiento actual de los sofocos inducidos por deprivación androgénica en el carcinoma prostático
}

\author{
Santiago Vilar González*, Francesc Montañá Puig**, Manuel Aguayo Martos*, \\ $\mathrm{M}^{\mathrm{a}}$ Victoria Villas Sánchez*, $\mathrm{M}^{\mathrm{a}}$ del Mar Sevillano Capellán*, Sebastiá Sabater Martí*.
}

${ }^{*}$ Complejo Hospitalario Universitario de Albacete (CHUA). ${ }^{* *}$ Angelini-Farmaceútica.

\section{Resumen}

Dada la mayor incidencia, prevalencia y supervivencia del carcinoma prostático en la actualidad, el manejo de los sofocos derivados de su tratamiento con análogos LH-RH ha de ser muy tenido en cuenta.

El tratamiento más utilizado y a la vez el más eficaz, es la sustitución hormonal pero este tipo de terapia no esta exenta de riesgos. Hoy por hoy es factible el abordaje de los sofocos de estos pacientes mediante un variado arsenal terapéutico en el cual el tratamiento hormonal puede quedar relegado al último lugar, dado el riesgo de recidiva o progresión tumoral al tratarse de un tumor hormonosensible.

El objetivo de este trabajo es revisar los tratamientos utilizados actualmente y las medidas higiénico-dietéticas que pueden ayudar a disminuir la sintomatología.

Se revisarán tanto los tratamientos hormonales como los no hormonales basados en su evidencia científica.

Fármacos como los nuevos antidepresivos, la gabapentina y la clonidina podrían jugar un papel destacado en el manejo. Sus mecanismos de actuación aunque dispares, se enmarcan en el complejo sistema de retroalimentación ejercido por los niveles de hormonas sexuales sobre la secreción hipotalámica de noradrenalina, causa principal en la génesis de los sofocos.

Palabras clave: Carcinoma prostático. Sofocos. Deprivación hormonal. Tratamiento. Antidepresivos. Clonidina. Gabapentina. Tratamiento hormonal sustitutivo.

\section{Review of current treatment for hot flushes induced by androgen deprivation in prostate carcinoma}

\section{Abstract}

Considering the currently increased incidence, prevalence and survival of prostate cancer, the management of hot flushes associated with $\mathrm{LH}-\mathrm{RH}$ analog treatment must be taken into account.

The most widely used and effective treatment is hormone replacement, though the latter is not without risks. It is presently possible to address hot flushes in these patients based on a broad range of treatment options in which hormone therapy may constitute a last option, due to the risk of tumor relapse or progression - since prostate cancer is hormone sensitive.

The present study reviews the currently used treatments and hygiene-dietary measures that may help reduce the symptoms.

A review is made of both hormone and non-hormone therapies, based on the existing scientific evidence.

Drugs such as the new antidepressants, gabapentin and clonidine may play an important role in the management of hot flushes. While the underlying mechanisms of action are varied, they are related to the complex feedback exerted by the sexual hormones upon the hypothalamic secretion of noradrenalin - this being the principal etiological factor of hot flushes.

Keywords: Prostate carcinoma. Hot flushes. Hormone deprivation. Treatment. Antidepressants. Clonidine. Gabapentin. Hormone replacement therapy.

$\mathrm{D}$ ebido a la alta incidencia de carcinoma prostático en los países desarrollados sumado al hecho de que en muchos de estos pacientes la terapia con deprivación androgénica jugará un papel importante en su tratamiento, hace que cada día adquiera más relevancia el correcto manejo de la toxicidad provocada.

El mecanismo más utilizado en la actualidad para dicha deprivación son los análogos LH-RH, en conjunción o no con los antiandrógenos.

Estudios previos demostraron su igualdad de eficacia en relación a la orquidectomia quirúrgica ${ }^{1,2}$.
Cabe destacar que se ha observado una mayor presencia de sofocos-sudores en el grupo tratado con análogos (60-75\%) $)^{3-8}$. En la terapia con análogos los sofocos se pueden presentar durante varios meses pudiendo persistir aunque en menor intensidad y frecuencia durante años.

La descripción de los sofocos se la debemos a Cabot que estudió los efectos de la castración en el tratamiento del carcinoma prostático ${ }^{9}$.

Los sofocos pueden ir acompañados de multitud de otros síntomas y signos como rubefacción, palpi- 
taciones, ansiedad, y sensación de pérdida de control, con gran repercusión física y emocional, influenciando en gran medida en detrimento de la calidad de vida de estos pacientes ${ }^{6,10,11}$.

$\mathrm{Su}$ adecuado control es de vital importancia pero destacando que tumores hormonosensibles no deberían ser tratados con terapia hormonal, a pesar haber demostrado mayor eficacia, hasta que estudios controlados con adecuada metodología y mayores seguimientos pongan en evidencia la ausencia de riesgo oncológico ${ }^{12,13}$.

El motivo del presente trabajo es revisar el amplio arsenal terapéutico del que disponemos en la actualidad para el manejo de esta sintomatología.

Con tal finalidad y tras una búsqueda bibliográfica inicial en PubMed, se han analizado los trabajos resultantes. Los propios artículos obtenidos han servido de base para ampliar dicha búsqueda hasta obtener todos los manuscritos originales de interés.

\section{TRATAMIENTO ACTUAL DE LOS SOFOCOS}

Como cabría esperar, la gran mayoría de estudios publicados se han llevado a cabo en mujeres menopáusicas o en mujeres supervivientes a un carcinoma mamario.

En vista a su fisiopatogénesis se deben tener en cuenta los mecanismos reguladores sobre la retroalimentación negativa (feedback) como causantes de dichos sofocos ${ }^{13}$.

Actualmente se considera como causa principal de dicha sintomatología la deprivación hormonal abrupta con intervención hipotalámica ${ }^{13}$. La proximidad del centro termorregulador hipotalámico con las zonas productoras de LH-RH intervendría en el problema $^{14-16}$.

Los opioides endógenos también estarían implicados en el proceso ${ }^{17}$. Las $\beta$-endorfinas se ven incrementadas por las hormonas sexuales periféricas, las cuales a su vez, producen catecolestrógenos causando disminución de la síntesis hipotalámica de $\mathrm{NA}^{7,15,18-20}$.

La caída abrupta de la carga hormonal va a producir una disminución de endorfinas y catecolestrógenos, por lo cual se van a incrementar los niveles de noradrenalina (NA) hipotalámica que al actuar sobre el centro productor LH-RH y por cercanía estimulando el centro termorregulador causará una disminución de los intérvalos de respuesta al estímulo calor-frío ${ }^{18,21}$. Otros neurotransmisores como serotonina y dopamina tienen un efecto contrario inhibiendo los sofocos ${ }^{18,19,22-27}$.

\section{REEMPLAZO HORMONAL}

El reemplazo hormonal con estrógenos fue el primer tratamiento utilizado en el siglo XX. Otras formas de reemplazo incluían progestágenos y andrógenos; pero dada la hormonosensibilidad del cáncer de próstata estos últimos estarían contraindicados.

En cuanto a los estrógenos, indicar que a pesar de su elevada eficacia incluso a baja dosis; presentan efectos secundarios negativos. Entre ellos caben destacar los fenómenos tromboembólicos, morbilidad cardiovascular y ginecomastia dolorosa $a^{28-30}$.

El estrógeno más utilizado, tanto vía oral como transdérmica, ha sido el estradiol ${ }^{19,31}$, necesitándose un tratamiento de al menos un mes para obtener beneficios. A mayores dosis mayor control, pero también mayores efectos secundarios siendo menores en el caso de uso de presentaciones transdérmicas.

Las dosis habituales vía oral son de $\geq 0,25$ mg/día mientras que para los parches la cantidad diaria necesaria es de al menos 0,05 mg/día.

En resumen la terapia estrogénica presenta una respuesta dosis dependiente, con un balance a favor de la terapia transdérmica y una eficacia del 80-90 $\%$, reduciendo de 2,5 a 3 los episodios de sofocos diarios ${ }^{14,28,32}$, pero con unos efectos secundarios indeseables a tener en cuenta ${ }^{13,14,33,34}$.

Su efectividad ha sido constatada en un metaanalisis de la Librería Cochrane del año 2002, y en una extensa revisión publicada en JAMA en el año $2004^{32}$.

Los progestágenos al igual que los estrógenos estimulan la producción hipotalámica de $\beta$-endorfinas. Su uso tampoco está exento de efectos secundarios indeseables ${ }^{21,35,36}$. Existe experiencia tanto en hombres como en mujeres siendo los más utilizados el acetato de megestrol con dosis iniciales de $20 \mathrm{mg}$ cada 12 horas y posterior reducción de dosis hasta la mínima eficaz, con tasas de respuesta del 80-90\% ${ }^{3,13,21,28,35,37}$.

Como segundo fármaco en uso estaría el acetato de ciproterona, un antiestrógeno esteroideo con efecto progestagénico; con tasas de respuesta comparables al anterior pero con riesgo de hepatotoxicidad, fatiga, ginecomastia dolorosa y galactorrea. Se indica iniciar el tratamiento con $50 \mathrm{mg} /$ día, no debiendo superar los $300 \mathrm{mg} /$ día $^{13,38}$.

En último lugar hay que hablar del acetato de medroxiprogesterona ${ }^{39-41}$ a dosis de 20-40 mg al día vía oral con igual o superior eficacia que el megestrol $^{42,43}$ o $400 \mathrm{mg}$ IM en sistema depot con dosis 
única y efecto mantenido en el tiempo de al menos 6 meses $^{39,43}$. Aunque algunos expertos consideran $400 \mathrm{mg}$ como una dosis muy elevada, se trata de una dosis pequeña si se compara con los 500 mg IM o vía oral utilizados diariamente durante meses para el tratamiento del cáncer de mama ${ }^{39,43,44}$, demostrándose una correcta tolerancia, con aumento de peso como único efecto indeseable ${ }^{44}$.

El uso de progestágenos en el cáncer de próstata presenta algunas reservas al describirse en algunos artículos su relación con incrementos en los niveles de PSA $^{45-47}$. Extrapolar estos resultados al uso de bajas dosis de progestágenos en el tratamiento del cáncer de próstata no queda aclarado ${ }^{39,48}$, aunque también se ha descrito su actividad antitumoral en cáncer de mama ${ }^{49}$, endometrio ${ }^{50}$ y próstata $^{51}$.

$\mathrm{El}$ efecto de los estrógenos y progestágenos puede perdurar en el tiempo tras la retirada de los $\operatorname{mismos}^{21,52}$.

Los problemas de la utilización del reemplazo hormonal en mujeres menopáusicas también ha adquirido cierto protagonismo a raíz de la publicación de los trabajos del Women's Health Initiative randomized controlled Trial, que pone en evidencia el mayor riesgo de desarrollo de cáncer de ma$\mathrm{ma}^{53,54}$, trastornos cognitivos, enfermedad cardiovascular y tromboembólica ${ }^{33,34,39}$ siendo por ello deseables otros tratamientos alternativos. El riesgo absoluto por 10.000 personas/año es 7 veces mayor para eventos coronarios, 8 más en AVC (accidente vascular cerebral), 8 más para TEP (trastorno embólico periférico), y 8 más para carcinoma invasivo mamario; mientras que el beneficio aportado es de 6 cánceres colorectales menos y 5 fracturas de cadera. Asimismo también reduce los fenómenos vasomotores y la atrofia vulvovaginal ${ }^{54}$.

\section{TRATAMIENTOS NO HORMONALES}

Resulta interesante indicar que existen otros tratamientos no hormonales. Esto es de suma importancia tanto para las mujeres supervivientes de un cáncer de mama, como para los hombres afectos de un adenocarcinoma prostático con deprivación hormonal, donde las repercusiones del tratamiento hormonal sustitutivo pueden contraindicar su uso. (Tabla 1).

\section{Tabla 1}

Listado de medicamentos

Estrógenos $\quad$ Estradiol v.o $(0,25 \mathrm{mg} /$ día) o transdérmico $(0,05 \mathrm{mg} /$ día $)$

Progestágenos: Acetato de megestrol $20 \mathrm{mg} / 12 \mathrm{~h}$

Acetato de ciproterona $50 \mathrm{mg} /$ día

Acetato de medroxiprogesterona v.o 20-40 mg/día o depot i.m $400 \mathrm{mg}$ dosis única
$\%$ de control

80-90

$80-90$

Clonidina de 25 a 400 microg/día v.o

60-65

55-65

Venlafaxina 25-75 mg/día v.o

Sertralina $50 \mathrm{mg} /$ día v.o

Trazodona 50-150 mg/día v.o

Citalopram

Veraliprida

Meclobemida

Gabapentina 300 mg/8 h v.o Incluso eficiaz tras fallo de los ADT

Efecto placebo

Otros que no han demostrado su eficacia:

Bellegard

Isoflavonas (fitoestrógenos)

Vit E

Cimifuga racemosa

Acupuntura

Metildopa

Ejercicio físico

Técnicas de relajación 
Por lo que respecta a los fenómenos vasomotores son muchos los fármacos estudiados para tal fin. Un reciente metaanalisis del $2006^{55}$ pone en evidencia que las únicas terapias efectivas sobre la base de la bibliografía existente, con estudios metodológicos válidos, son en orden decreciente según su efectividad: la gabapentina, los inhibidores selectivos de la recaptación de serotonina y la clonidina.

A continuación los describiremos de manera somera. Su eficacia no parece ser igual a la sustitución hormonal pero si han demostrado ampliamente su efectividad ${ }^{14,39,56}$.

Ya que la noradrenalina es el neurotransmisor implicado directamente en el control del centro termoregulador, el bloqueo de los adrenoreceptores tanto alfa como beta pueden controlar la clínica. Partiendo de este razonamiento se ha venido utilizando tanto en hombres ${ }^{8,27}$ como en mujeres $^{57-59}$, la clonidina; un a2 agonista ${ }^{57}$. Se han encontrado estos receptores (a2) tanto a nivel hipotalámico como periférico. Su activación presináptica se traduce en una reducción de la liberación de $\mathrm{NA}^{60}$. Asimismo presenta acción periférica reduciendo la vasodilatación y contribuyendo por ello a un mayor control de los sofocos ${ }^{13}$. A mayor dosis mayor control, así como mayor toxicidad. Su administración transdérmica ${ }^{61,62}$ es equiparable a su administración oral ${ }^{59,61-63}$ pero presenta frecuentes reacciones cutáneas. Las dosis recomendadas van de 25 a 400 $\mu \mathrm{g}$. Su eficacia es inferior a la terapia hormonal (20$55 \%)$ y se observa un alto porcentaje de efectos secundarios indeseables aunque dos recientes ensayos a doble ciego comparándola con la venlafaxina, con resultados dispares remarcan su buena tolerancia e incluso eficacia en el control de los sofo$\cos ^{64,65}$. Su indicación puede ser contemplada ante la contraindicación al uso de otras terapias ${ }^{13}$.

Se ha utilizado la gabapentina a dosis de 300 $\mathrm{mg} / 8 \mathrm{~h}$, con respuestas del 44-60\%, describiéndose: somnolencia, mareos, fatiga, erupción cutánea, palpitaciones y edema periférico ${ }^{66-69}$. Su actuación potencial sería a través de la modulación de los canales de calcio, aunque hoy por hoy continua sin aclararse su mecanismo de acción concreto ${ }^{69}$. En un estudio reciente a demostrado su utilidad tras la no adecuada respuesta a los antidepresivos ${ }^{68}$.

Por lo que respecta al potencial uso de determinados antidepresivos y a su efecto sobre la serotonina, éstos se presentan como fármacos de interés. Se sabe que los niveles de serotonina en mujeres postmenopausicas están disminuidos; y tras terapias sustitutivas, éstos parecen tender a normalizarse. En base a ello, una disminución abrupta de hormonas sexuales produciría una reducción en la circulación de serotonina, con el consiguiente aumento de sus receptores $5-\mathrm{HT}_{2 \mathrm{~A}}$ hipotalámicos ${ }^{22,24}$ los cuales estarían implicados en la patogénesis de los sofocos ${ }^{22}$.

La potencia del bloqueo de los receptores de serotonina $5-\mathrm{HT}_{2 \mathrm{~A}}$ varía considerablemente entre los antidepresivos de primera generación (tricíclicos). La importancia de dicho efecto sobre la acción terapéutica de los antidepresivos tricíclicos en general no está clara. Sin embargo, existe otra clase de antidepresivos, conocidos como fenilpiperazinas, que son más selectivos que los antidepresivos tricíclicos y cuya acción farmacológica más potente es la de bloquear a los receptores $5 \mathrm{HT}_{2 \mathrm{~A}}{ }^{70}$.

Nuevos antidepresivos, en especial la venlafaxina, la paroxetina y en menor medida la fluoxetina, podrían desempeñar un importante papel en la terapia no hormonal de los sofocos ${ }^{48,64,65,71-74}$, con respuestas en torno al 50-65\%, algo menores que las terapias hormonales pero con un perfil de seguridad mayor en los supervivientes de cáncer que los hace muy interesantes para este tipo de pacientes como alternativa $^{22}$. Sus efectos secundarios, aunque presentes, no son de gran relevancia al utilizarse dosis menores de las habituales para el tratamiento de las depresiones y no haber presentado síndrome de abstinencia a tales dosis ${ }^{22,64,65}$ (Tabla 2).

Tabla 2. Dosis y tasas de respuesta de algunos antidepresivos

- Paroxetina a dosis entre 10 y $25 \mathrm{mg} /$ día con reducciones de $60-65 \% 16,71,82$.

- Venlafaxina dosis de 25 o de 37,5 en pauta ascendente en una semana hasta $75 \mathrm{mg} /$ día, con tasas de respuesta del 50-65 \% $48,64,65,73,83-85$.

- Fluoxetina a $20 \mathrm{mg} /$ día con efectividad en torno al $50 \%{ }^{72,74}$.

- Citalopram ${ }^{72}$.

- Veraliprida (antidopaminérgico) ${ }^{33}$.

- Sertralina a $50 \mathrm{mg} /$ día con respuestas del $36 \%^{86}$.

Son escasas las publicaciones que hemos encontrado sobre el uso de antidepresivos de nueva generación en el tratamiento de los sofocos en el paciente oncológico prostático sometido a deprivación hormonal. Un estudio con venlafaxina en 16 pacientes muestra que el fármaco parece ali- 
viar la sintomatología ${ }^{48}$. Está también disponible un estudio piloto con paroxetina que incluye 24 pacientes, apuntándose un alivio de las crisis de sofocos en el hombre ${ }^{71}$.

Como dato de interés, indicar que el efecto placebo puede controlar los sofocos en un 20-40\% de los pacientes, con percepciones individuales de mejoría de hasta un 50-75 \% sobre los síntomas iniciales $^{48,75-77}$.

Se han utilizado otros muchos productos sin tanta efectividad, nula efectividad o desestimados por la elevada incidencia de sus efectos secundarios (Tabla 3).

Por otra parte resulta provechoso recomendar medidas higiénico-dietéticas para prevenir los sofocos como: lavados con agua fría o aplicación de frío, no comer ni beber alimentos demasiado calientes, no permanecer en ambientes muy calurosos, limitar la ingesta de especias, café y alcohol, realizar una dieta rica en productos que contengan proteínas de la soja (y no fitoestrogenos como se preconiza) ${ }^{77,78}$, reducir el estrés mediante técnicas de relajación, realizar ejercicio físico no vigoroso para mantener un peso adecuado (si es excesivo puede aumentar la frecuencia e intensidad de los sofocos) 18 y abandonar el hábito tabáquico ${ }^{76}$ (Tabla 3).

Recientes investigaciones han demostrado una correlación entre el índice de masa corporal y la frecuencia de los sofocos, la cual vendría condicionada por el metabolismo insulínico en la grasa y por un aumento de la temperatura corporal ${ }^{18,78,79}$. Asimismo se ha relacionado con el hábito tabáquico, posiblemente a través del efecto sobre el metabolismo estrogénico o por el efecto termogénico de la nicotina ${ }^{18,80,81}$.

Tabla 3. Algunas medidas empleadas para controlar los sofocos

- Bellergal (fenobarbital + ergotamina + alcaloides de la belladona) con un $30 \%$ de abandono de la terapia por toxicidad $^{87}$

- Suplementos dietéticos ${ }^{77}$.

- Suplementos a altas dosis de isoflavonas/fitoestrógenos con nulo efecto (presentando tanto efecto estrogénico como antiestrogénico ${ }^{88}$.

- Plantas medicinales ${ }^{77}$.

- Vit $\mathrm{E}^{77}$.

- Técnicas de relajación ${ }^{88}$.

- Ejercicio físico 88 .

- Metildopa ${ }^{89,90}$.

- Acupuntura ${ }^{91-93}$.

\section{CONCLUSIÓN}

Son frecuentes los sofocos en los pacientes sometidos a terapia antiandrogénica por carcinoma prostático. La asociación entre cáncer de próstata, deprivación androgénica y sofocos es evidente, repercutiendo de manera intensa en la calidad de vida de estos pacientes.

El uso de terapia sustitutiva hormonal, tanto con estrógenos como con progestágenos viene dificultada por la hormonodependencia de la patología que nos ocupa, lo que hace necesario buscar alternativas terapéuticas que puedan ser útiles en determinados casos.

Los tratamientos no hormonales no parecen tener una eficacia similar a la terapia hormonal. Se han preconizado medidas higiénico-dietéticas $\mathrm{y}$ también se ha relacionado la obesidad, por su implicación con el metabolismo insulínico.

La clonidina, con una eficacia entorno al 20-55\% con respecto a la terapia hormonal, se ha planteado como un eficaz reductor de la liberación de NA. El efecto de modulación de la gabapentina sobre los canales del calcio parece actuar positivamente en la sintomatología, aunque el mecanismo de acción no esté aún bien evidenciado.

Es sabido que la disminución abrupta en la circulación de hormonas sexuales reduce los niveles de serotonina determinando un incremento en el número de receptores $5-\mathrm{HT}_{2 \mathrm{~A}}$ hipotalámicos implicados en la fisiopatología de los sofocos.

Los antidepresivos serotoninérgicos pueden desempeñar un papel importante al incrementar la serotonina a nivel central, por lo que pueden causar un balanceo a la NA en el centro termorregulador.

Se han ensayado antidepresivos serotoninérgicos en pacientes masculinos bajo deprivación hormonal, tipo venlafaxina, paroxetina y fluoxetina con resultados positivos aunque algo menores que los obtenidos con terapias hormonales sustitutivas, en torno al 50-65 \%; pero con un perfil de seguridad mucho mayor.

Serían necesarios mas ensayos clínicos siguiendo estas líneas que clarifiquen el lugar que pueden jugar todas estas terapia en el tratamiento de los sofocos inducidos por la deprivación androgénica con análogos en el carcinoma prostático. Es un tema de máximo interés y actualidad debido a la mayor incidencia de esta patología en nuestra actual sociedad, con el detrimento en la calidad de vida de nuestros pacientes y su entorno por ello ocasionado. 


\section{REFERENCIAS}

1. Parmar H, Edwards L, Phillips RH, Allen L, Lightman SL. Orchidectomy vs long acting D-Trip-6-LHRH in advanced prostate cancer. Br J Urol. 1987;59(3):248-254.

2. Béland G, Elhilali M, Fradet Y, et al. Total androgen blockade vs castration in metastatic cancer of the prostate. Hormonal Therapy of prostatic Disease: Basic and Clinical Aspects. 35. Proceeding of an International Symposium. Milan, Italy April 6-8. Medicom, 1987;243A:391-400.

3. Charig CR, Rundle JS. Flushing. Long-term side effects of orchidectomy in treatment of prostatic cancer. Urology. 1989;33 (3):175-178.

4. Buchholz NP, Matarelli G. Hot flushes after orchidectomy in treatment of prostate cancer - a serious effect. Z Gerontol. 1994;27(5):334-336.

5. Sarosdy MF, Schellhammer PF, Soloway MS et al. Endocrine effects, efficacy and tolerability of a 10,8 mg depot formulation of goserelin acetate administered every 13 weeks to patients with advanced prostate cancer BJU Int, 1999;83(7):801-806.

6. Nishiyama T, Kanazawa S, Watanabe R, Terunuma M, Takahashi K. Influence of hot flashes on quality of life in patients with prostate cancer treated with androgen deprivation therapy. Int J Urol. 2004;1 1(9):735-741.

7. Holdaway IM, Ibbertson HK, Croxson MS, et al. Treatment of metastatic prostate cancer with depot LH-RH analogue Zoladex. Prostate. 1988;12(2)119-127.

8. Schow DA, Renfer LG, Rozanski TA, Thompson IM. Prevalence of hot flushes during and after neoadjuvant hormonal therapy for localized prostate cancer. South Med J. 1998;91(9):855-857.

9. Cabot AT. The question of castration for enlarged prostate. Am Surg, 1896;24(3):265-309.

10. Kronenberg Fl. Hot flashes: phenomenology, quality of life and search for treatment options. Exp Gerontol. 1994; 29 (3-4): 319-336.

11. Ginsburg J. O'Reilly B. Climateric flusing in a man. Brit Med J, 1983; 287(6387):262.

12. Smith JA. Management of hot flushes due to endocrine therapy for prostate cancer. Oncology. 1996;10:1319-1322.

13. Kouriefs C, Georgiou M, Ravi R. Hot flushes and prostate cancer: pathogenesis and treatment. BJU International. 2002;89(4):379-383.

14. Rossouw JE, Anderson GL, Prentice RL, et al. Risks and benefits of estrogen plus progestin in healthy postmenopausal women: principal results from the Women's Health Initiative randomized controlled Trial. JAMA 2002(2);288: 321-333.

15. Freedman RR, Krell W. Reduced thermorregulatory null zone in postmenopausal women with hot flashes. Am J Obstet Gynecol. 1999;18(1):6670.

16. Stearns V, Johnson MD, Rae JM, et al. Paroxetine controlled release in the treatment of menopausal hot flashes: a randomized controlled trial. JAMA. 2003;289(21):2827-2834.

17. Stubbs WA, Delitala G, Jones A, et all. Hormonal and metabolic responses to an enkephalin analogue in normal man. Lancet. 1978; 2 (8102): 1225-1227.

18. Robert R. Hot flashes: behavioral treatments, mechanims, and relation to sleep. Am J Med, 2005;118:124S-30S.

19. Shanafelt TD, Barton DL, Adjei AA, Loprinzi CL. Pathophysiology and treatment of hot flashes. Mayo Clin Proc. 2002;77(11):1207-1218.

20. Fishman J, Norton BL, Hahn EF. Opiate regulation of estradiol-2 hydroxylase in the brain of female rats: Mechanism for control of pituitary hormone secretion. Proc Natl Acad Sci USA, 1980;77(3):2574-2576.

21. Loprinzi CL, Michalak JC, Quella SK, et al. Megestrol acetate for the prevention of hot flushes. N Engl J Med, 1994;331(16):347-352.

22. Albertazzi P. Nonadrenergic and serotonergic modulation to treat vasomotor symptoms. J Br Menopause Soc. 2006;12(1):7-11.

23. Freedman RR. Physiology of hot flashes. Am J Hum Biol, 2001;13(4): 453-464.

24. Curcio JJ, Kim LS, Wollner D, Pockaj BA. The potential role of 5Hydroxytryptophan for hot flash reduction: A hypothesis. Altern Med Rec, 2005;10(3):216-221.

25. Kalay AE, Demir B, Haberal A, Kalay M, Kandemir O. Efficacy of citalopram on climacteric symptoms. Menopause. 2007;14 (2):223-229.

26. Berendsen HH. The role of serotonine in hot flushes. Maturitas, 2000; 36 (3): $155-164$

27. Freedman RR. Pathophysiology and treatment of menopausal hot flashes. Semin Reprod Med. 2005;23(3):117-125.
28. Smith JA. A prospective comparison of treatments for symptomatic hot flushes following endocrine therapy for cancer of the prostate. J Uro. 1994;152:132-413.

29. Miller JI, Ahmamn et al. Treatment of castration-induced menopausal symptoms with low dose diethylstilbestrol in men with advanced prostate cancer. Urology. 1992;40:499-502.

30. Atala A, Amin M, Harty JI. Diethylstilbestrol in treatment of postorchiectomy vasomotor symptoms and its relation-ship with serum folliclestimulating hormone, luteinizing hormone, and testosterone. Urology, 1992;39(1):108-110.

31. Savage MV, Brengelman GL. Control of skin blood flow in the neutral zone of human body temperature regulation. J Appl Physiol. 1996;80(4): 1249-1257.

32. Nelson HD. Commonly used types of postmenopausal estrogen for treatment of hot flashes: scientific review. JAMA. 2004;291(3):1610-1620.

33. Casper RF, Yen SS. Neuroendocrinology of menopausal flushes: a hypothesis of flush mechanism. Clin Endocrinol (Oxf), 1985;22 3):293-312.

34. Stearns V, Slack R, Greep N, et al. Paroxetine is an effective treatment for hot flashes: results from a prospective randomized clinical trial. $\mathrm{J}$ Clin Oncol. 2005;23(33):6919-6930.

35. Quella SK, Loprinzi CL, Sloan JA, et al. Long-term use of megestrol acetate by cancer survivors for the treatment of hot flashes. Cancer, 1998;82(2):1784-1788.

36. Loprinzi CL, Johnson PA, Jensen M. Megestrol acetate for anorexia and cachexia. Oncology. 1992;49:Suppl 2:46-49.

37. Goodwin JW, Green SJ, Moinpour CM, Bearden JD III, Giguere JK, Jian CS, Lippman SM, Martino S, Albain KS. . Phase III randomized placebocontrolled trial of two doses of megestrol acetate as treatment for menopausal symptoms in women with breast cancer: Southwest Oncology Group Study 9626. JCO, 2008;26:1650-56.

38. Moon TD. Cyproterone acetate for treatment of hot flushes after orchidectomy. Letter. J Urol. 1985;134(1):155-156.

39. Prior JC, McKay DW, Vigna YM, Barr SI. Medroxyprogesterone increases basal temperature: a placebo-controlled crossover trial in postmenopausal women. Fertil Steril. 1995;63(6):1222-1226.

40. Ronzoni G, De Giovanni L, Menchinelli P, Meneschincheri M.Therapy of vasomotor syndrome in the treatment of advanced prostatic cancer: apropos of 37 cases. Arch Ital Urol Androl. 1998;70(1):37-40.

41. Finck G, Barton DL, Loprinzi CL, Quella SK, Sloan JA. Definitions hot flashes in breast cancer survivors. J Pain Symptom Manage. 1998; 16(5):327-333.

42. Borm GF, Hoogendoorn EH, den Heijer M, Zielhuis GA. Sequential balancing: a simple method for treatment allocation in clinical trials. Contemp Clin Trials. 2005 Dec;26(6):637-645.

43. Benghiat A, Cassidy SA, Davidson HE, Mancero FS, Pickard JG, Tyrrell CJ. Megestrol acetate in the treatment of advanced post-menopausal breast cancer. Eur J Surg Oncol. 1986;12(1):3-45.

44. Shears P. Climacteric flushing in a man. Br Med J (Clin Res Ed). 1983; 287(6389):422-433.

45. Steinfeld $\mathrm{AD}$, Reinhardt $\mathrm{Cl}$. Male climateric after orchiectomy in patients with prostatic cancer. Urology. 1980;16(6):620-622.

46. Dawson N, McLeod DG. Dramatic prostate specific antigen decrease in response to discontinuation of megestrol acetate in advanced prostate of cancer: expansion of the antiandrogen withdrawal syndrome. J Urol 1995; 153(6): 1946-1947.

47. Wehbe TW, Stein BS, Akerley WL. Prostate-specific antigen response to withdrawal of megestrol acetate in a patient with hormone-refractory prostate cancer. Mayo Clin Proc. 1997;72(10):932-934.

48. Quella SK, Loprinzi CL, Sloan J, et al. Pilot evaluation of venlafaxine for the treatment of hot flashes in men undergoing androgen ablation therapy for prostate cancer. Journal of Urology. 1999;162(1):98-102.

49. Leuprolide Study Group. Leuprolide vs diethylstilbestrol for metastatic prostate cancer. N Engl J Med. 1984;311:1281-1286.

50. Wentz WB et al. Progestin therapy in lesions of the endometrium. Semin Oncol. 1985;12:Suppl 1:23-27.

51. Bonomi P, Pessis D, Bunting N, et al. Megestrol acetate used as primary hormonal therapy in stage D prostatic cancer. Semin Oncol. 1985;12(1): 36-39.

52. Haas S et al. The effect of transdermal estradiol on hormone and metabolic dynamic over a six-week period. Obstet gynecol. 1988; 71:671-676 
53. Haslam SZ, Osuch JR, Raafat AM, Hofseth LJ. Postmenopausal hormone replacement therapy: effects on normal mammary gland in humans and in a mouse postmenopausal model. J Mammary Gland Biol Neoplasia. 2002;7(1):93-105.

54. Anderson GL, Limacher M, Assaf AR, et al. Women's Health Initiative Steering Committee. Effects of conjugated equine estrogen in postmenopausal women with hysterectomy: Women's Health Initiative randomized controlled Trial. JAMA. 2004;291(14):1701-1712.

55. Nelson HD, Vesco KK, Haney E, et al. Nonhormonal therapies for Menopausal hot flashes. Systematic Review and Meta-analysis. JAMA, 2006; 295 (17): 2057-2071.

56. Lebherz TB, French L. Nonhormonal treatment of the menopausal syndrome. A double-blind evaluation of an autonomic system stabilizer. Obstet Gynecol. 1969; 33 (6): 795-799.

57. Clayden JR et al. Menopausal flushing. Double-blind trial of a non-hormonal medication. Br Med . 1974;1:409-412.

58. Ravnikar V. Physiology and treatment of hot flushes. Obstet Gynecol. 1990;75:3S-7S

59. Pandya KJ, Raubertas RF, Flynn PJ, et al. Oral clonidine in postmenopausal patients with breast cancer experiencing tamoxifen-induced hot flashes: a University of rochester cancer center Community Clinical Oncology Program study. Ann Intern Med. 2000;132 (10):788-793.

60. Freedman RR, Dinsay R. Clonidine raises the sweating threshold in symptomatic but not in asymptomatic postmenopausal women. Fertil Steril, 2000; 74 (1): 20-23.

61. Nagamani M, Hannigan EV, Dinh TV, Stuart CA. Treatment of menopausal hot flashes with transdermal administration of clonidine. Am J Obstet Gynecol. 1987;156(1):561-565.

62. Wren BG, Brow Lb. A double-blind trial with clonidine and a placebo to treat hot flushes. Med J Aust.1986;144(7)369-370.

63. Quella S, Loprinzi CL, Doge AM. A qualitive approach to defining " hot flashes " in men. Urol Nurs. 1994;14(3):155-158.

64. Buijs C, Mom CH, Willemse PH, Marike Boezen H, Maurer JM, Wymenga AN, de Jong RS, Nieboer P, de Vries EG, Mourits MJ. Venlafaxine versus clonidine for the treatment of hot flashes in breast cancer patients: a double-blind, randimized cross-over study. Breast cancer res Treat. 2008 Aug 1. (Epub ahead of print).

65. Loibl S, Schwedler K, Von Minckwitz G, Strohmeier R, Mehta KM, Kaufmann M. Venlafaxine is superior to clonidine as treatment of hot flashes in breast cancer patients - a double-blind, randomized study. Ann Oncol. 2007;18:689-693.

66. Loprinzi CL, Kugler JW, Barton DL, et al. Phase III trial of gabapentin alone or in conjunction with an antidepressant in the management of hot flashes in women who have inadequate control with an antidepressant alone: NCCTG N03C5. J Clin Oncol, 2007;25(3):308-312.

67. Pockaj BA, Gallagher JG, Loprinzi CL, et al. Phase III double-blind, randomized, placebo-controlled crossover trial of black cohosh in the management of hot flashes: NCCTG Trial N01CC. J Clin Oncol. 2006;24(18): 2836-2841.

68. Loprinzi L, Barton DL, Sloan JA, et al. Pilot evaluation of gabapentin for treating hot flashes. Mayo Clin Proc. 2002;77(11):1159-1163.

69. Guttuso TJ. Gabapentin's effects on hot flashes and hypotermia. Neurology. 2000;54(11):2161-2163.

70. Stahl SM. Nuevos antidepresivos y estabilizadores del estado de ánimo. Psicofarmacología esencial. $2^{\text {a }}$ ed. 2002; p.267-326.

71. Loprinzi Cl, Barton DL, Carpenter LA et al. Pilot evaluation of paroxetine for treating hot flashes in men. Mayo Clin Proc. 2004;79:1247-1251.

72. Suvanto-Luukkonen E, Koivunen R, Sundström H et al. Citalopram and fluoxetine in the treatment of postmenopausal symptoms: a prospective, randomized, 9-month, placebo-controlled, double-blind study. Menopause. 2005; 12(1):18-26.

73. Loprinzi CL, Kugler JW, Sloan JA, et al. Venlafaxine in manegement of hot flashes in survivors of breast cancer: a randomized controlled trial: Lancet. 2000;356(9247):2059-2063.

74. Loprinzi CL, Sloan JA, Perez EA. Phase III evaluation of fluoxetine for treatment of hot flashes. J Clin Oncol. 2002;20(6): 1578-1583.

75. Sloan JA, Loprinzi CL, Novotny PJ, Barton DL, Lavasseur BI, Windschitl H. Methodological lessons learned from hot flash studies. J Clin Oncol. 2001;19(23):4280-4290.
76. Tzingounis VA, Aksu MF, Greenblatt RB. Estriol in the management of the menopause. JAMA. 1978;239(16):1638-1641.

77. Moyad MA. Complementary/alternative therapies for reducing hot flashes in prostate cancer patients: reevaluating the existing indirect data from studies of breast cancer and postmenopausal women. Urology, 2002; 59(suppl 1):20-33.

78. Albertazzi P, Pansini F, Bonaccorsi G, Zanotti L, Forini E, De Aloysio D1. The effect of dietary soy supplementation on hot flashes. Obstet Gynecol. 1998;91(11):6-11.

79. Gold EB, Sternfeld B, Kelsey JL, et al. R. Relation of demographic and lifestyle factors to symptoms in a multi-racial/ethnic population of women 40-55 years of age. Am J Epidemiol. 2000;152(5):463-473.

80. Jessen $\mathrm{AB}$, Toubro S, Astrup A. Effects of chewing gum containing nicotine and caffeine on energy expenditure and substrate utilization in men Am J Clin Nutr. 2003;77(6): 1442-1447.

81. Whiteman MK, Staropoli CA, Langenberg PW, McCarter RJ, Kjerulff KH, Flaws JA. Smoking, body mass and hot flashes in midlife women. Obstet Gynecol, 2003;101(12):264-272.

82. Stearns V, Slack R, Greep N, et al. Paroxetine is an effective treatment for hot flashes: results from a prospective randomized clinical trial. J Clin Oncol. 2005;23(28):6919-6930.

83. Loprinzi CL, Pisansky TM, Fonseca R, et al. Pilot evaluation of venlafaxine hydrochloride for the therapy of hot flashes in cancer survivors. J Clin Oncol. 1998;6(7):2377-2381.

84. Loprinzi CL, Levitt R, Barton D, et al. Phase III comparison of depomedroxyprogesterone acetate to venlafaxine for managing hot flashes: North Central Cancer Treatment Group Trial N99C7. J Clin Oncol. 2006;24(9):1409-1414.

85. Evans ML, Pritts E, Vittinghoff E, McClish K, Morgan KS, Jaffe RB. Management of postmenopausal hot flashes with venlafaxine hydrochloride: a randomized controlled trial. Obstet Gynecol, 2005;105(1):161 166.

86. Kimmick GG, Lovato J, McQuellon R, Robinson E, Muss HB. Randomized, double-blind, placebo-controlled, crossover study of sertraline (Zoloft) for the treatment of hot flashes in women with early stage breast cancer taking tamoxifen. Br J. 2006;12(2):114-122.

87. Bergmans MG, Merkus JM, Corbey RS, Schellekens LA, Ubachs JM. Effect of Bellergal Retard on climateric complaints: a double-blind, placebo-controlled study. Maturitas. 1987;9(3):227-234.

88. Loprinzi CL, Stearns V, Barton D. Centrally active nonhormonal hot flash therapies. Am J Med. 2005 19;118, Suppl12B:118-123.

89. Hammond MG, Hatley L, Talbert LM. A double blind study to evaluate the effect of methyldopa on menopausal vasomotor flushes. J Clin Endocrinol Metab, 1984;58(6):1158-1160.

90. Nesheim BI, Saetre T. Reduction of menopausal hot flashes by methyldopa: a double-blind crossover trial. Eur J Clin Pharmacol, 1981;20(6) 413-416.

91. Hammar M, Frisk J, Grimås O, Höök M, Spetz AC, Wyon Y. Acupuncture treatment of vasomotor symptoms in men with prostatic carcinoma: a pilot study. J Urol. 1999;161(1):853-856.

92. Towlerton G, Filshie J, O'Brien M, Duncan A. Acupuncture in the control of vasomotor symptoms caused by tamoxifen. Palliat Med. 1999;13 (5):445.

93. Wyon $Y$ et al. Acupuncture against climacteric disorders? Lower number of symptoms after menopause. Lakartidningen, 1994;91:2318-2322.

Correspondencia autor: Santiago Vilar González

Servicio de Radioterapia.

Complejo Hospitalario Universitario de Albacete (CHUA)

C/ Hnos. Falco, s/n - 02006 Albacete

Tef : 0034967597523

E-mail autor: santiagov06@gmail.com

Información artículo: Revisión - Cáncer de próstata

Trabajo recibido: diciembre 2008

Trabajo aceptado: enero 2008 